\title{
NOUVELLE
}

\section{Dysplasie bronchopulmonaire et génétique}

Alice Hadchouel, Christophe Delacourt

Service de pneumologie pédiatrique,

Hôpital Necker-Enfants Malades,

149-161, rue de Sèvres, 75015 Paris, France ;

Inserm U955, Créteil, France ; Fondation PremUP,

Paris, France.

alice.hadchouel-duverge@nck.aphp.fr
Héritabilité de la dysplasie bronchopulmonaire

Avec les progrès considérables de la réanimation néonatale et le contrôle optimal des différents facteurs de risque identifiés, l'incidence de la dysplasie bronchopulmonaire $(D B P)^{l}$ a dans un premier temps diminué pour atteindre un plateau, puis à nouveau augmenter depuis quelques années [1]. Pour expliquer cette stagnation épidémiologique, les études de concordance entre jumeaux confortent I'hypothèse d'une susceptibilité génétique à la DBP et suggèrent qu'il s'agit d'une maladie complexe, multifactorielle, résultant d'une combinaison de facteurs génétiques et d'effets environnementaux [2]. Le développement du poumon distal chez l'enfant prématuré serait ainsi dépendant d'interactions complexes entre l'environnement au sens large (in- ou ex-utero) et des gènes de susceptibilité, capables de moduler l'adaptation de l'enfant en développement à des conditions pathologiques. R.A. Parker et al. ont ainsi montré que le développement d'une DBP chez un jumeau était un facteur de risque très significatif de DBP chez l'autre jumeau, indépendamment de l'ordre de naissance et des autres facteurs confondants (OR ajusté : 12,3; $p<0,001$ ) [3]. Plus récemment, I'héritabilité a été estimée en comparant les concordances pour la DBP chez des paires de jumeaux monozygotes, partageant $100 \%$ de leur génome, et des paires dizygotes, partageant en moyenne $50 \%$ de leur génome. V. Bhandari et al. ont ainsi étudié 63 paires de jumeaux

\footnotetext{
${ }^{1}$ Pour définition, voir Encadré, page 827 de ce numéro.
}

monozygotes et 189 paires de jumeaux dizygotes et ont pu estimer l'héritabilité de la DBP à $53 \%$ après ajustement pour les variables de confusion [4]. Plus récemment, P.M. Lavoie et al. ont également calculé que l'héritabilité de la DBP dans une population de 318 jumeaux prématurés (70 paires monozygotes et 89 paires dizygotes) était de $82 \%$ [5]. Les différences de concordance entre jumeaux monozygotes et dizygotes pour la survenue d'une DBP sont observées lorsque l'on adopte pour définir la DBP le critère de persistance d'une oxygénothérapie à 36 SA, mais disparaissent lorsque I'on inclut les DBP légères, c'est-à-dire les enfants que l'on peut sevrer des besoins supplémentaires en oxygène entre le $28^{\mathrm{e}}$ jour et 36 SA [5]. Le phénotype clinique à $J 28$ ne serait donc que le reflet des agressions environnementales, alors que la persistance de l'oxygénodépendance à $36 \mathrm{SA}$ dépendrait d'interactions gènesenvironnement. De fait, une évaluation faite à 36 SA est bien mieux corrélée au devenir respiratoire à moyen et long terme que si elle est faite à 28 jours [6].

\section{Recherche de gènes de suceptibilité}

De nombreux gènes candidats pourraient être impliqués dans le risque de DBP, car ils interviennent dans la régulation du développement alvéolaire, la réponse inflammatoire, les défenses anti-oxydantes, les processus de réparation cellulaire après agression, ou encore les défenses antiinfectieuses [13, 14] $(\rightarrow)$.

$(\rightarrow)$ Voir les Nouvelles de $\varepsilon$ Lopez et P.H. Jarreau,

et de $\varepsilon$. Zana-Taïeb

et P.H. Jarreau, pages 823 et 826 de ce numéro
Plusieurs approches « gènes candidats 》 ont déjà été réalisées. Cependant, les études disponibles sont souvent limitées à un nombre réduit de patients et les résultats n'ont, pour la plupart, pas été reproduits dans d'autres cohortes. Les résultats de ces études "gènes candidats » sont résumés dans le Tableau I.

Il est peu probable que les études basées sur l'analyse d'un seul gène puissent réellement contribuer à l'identification des variants exerçant l'influence la plus significative. Les études d'association pangénomiques, qui utilisent des centaines de milliers de polymorphismes mononucléotidiques, augmentent considérablement notre capacité à identifier les influences génétiques de maladies complexes, multifactorielles. Une première étude de ce type a été conduite dans une population de 418 nouveau-nés prématurés issus de trois centres de réanimation néonatale d'île-de-France [7]. Ce travail a permis d'identifier le gène codant pour le protéoglycane de la matrice extracellulaire SPOCK2 (sparc/osteonectin, cwCV and kazal-like domains proteoglycan) comme un gène de susceptibilité très prometteur, bien que son rôle dans le développement du poumon n'ait pas encore été étudié [7]. Ce gène a été identifié par criblage pangénomique de deux populations ethniques différentes et le polymorphisme le plus significativement associé à la DBP a été validé par génotypage individuel dans deux populations différentes indépendantes, l'une française, issue des 3 centres participants, et l'autre finlandaise, avec des odds ratios $>2,5$. De plus, l'expression du gène SPOCK2 augmente 


\begin{tabular}{|c|c|c|c|}
\hline Gène & Polymorphisme & $\mathbf{n}$ & Susceptibilité à la DBP \\
\hline \multirow[t]{7}{*}{ TNF $\alpha$} & $-238 \mathrm{G} / \mathrm{A}$ & 100 & Allèle A protecteur \\
\hline & & 105 & Aucune \\
\hline & $-308 \mathrm{G} / \mathrm{A}$ & 100 & Aucune \\
\hline & & 178 & Aucune \\
\hline & & 105 & Aucune \\
\hline & $-857 \mathrm{C} / \mathrm{T},-863 \mathrm{C} / \mathrm{A},-1031$ & 105 & Aucune \\
\hline & $T / C$ & & \\
\hline \multirow[t]{5}{*}{ MBL2 } & R52C & 75 & Hétérozygote RC à risque \\
\hline & & 284 & Aucune \\
\hline & G54D & 284 & $G D$ et $D D$ à risque \\
\hline & & 99 & Aucune \\
\hline & G57દ & 99 & Aucune \\
\hline IL4 & Intron 3, promoteur & 224 & Aucune \\
\hline IL10 & $-1082 \mathrm{G} / \mathrm{A}$ & 294 & Aucune \\
\hline IL12 & p40 promoteur CTCTAA/GC & 153 & Aucune \\
\hline IL18 & Multiples SNP étudiés & 346 & Aucune \\
\hline $\mathrm{MCPl}$ & $-2518 \mathrm{~A} / \mathrm{G}$ & 178 & Aucune \\
\hline \multirow[t]{3}{*}{ TGF $\beta$} & $915 \mathrm{G} / \mathrm{T}$ & 178 & Aucune \\
\hline & $-800 \mathrm{G} / \mathrm{A}$ & 181 & Aucune \\
\hline & $915 \mathrm{G} / \mathrm{T}$ & 192 & Aucune \\
\hline IFN $\gamma$ & 874 T/A & 153 & Allèle T protecteur \\
\hline MIF & $-173 \mathrm{G} / \mathrm{C}$ & 103 & Allèle $C$ protecteur \\
\hline \multirow[t]{4}{*}{$S P-B$} & Plusieurs SNP intron 4 & 140 & Aucune \\
\hline & $\mathrm{i} 4 \Delta$ & 245 & Allèle $\Delta$ à risque \\
\hline & $B-18 \mathrm{~A} / \mathrm{C}$ & 71 & Allèle $\mathrm{C}$ à risque \\
\hline & Microsat. 6 AAGG & 71 & A risque \\
\hline SP-Al & $6 \mathrm{~A} 6$ & 46 & A risque \\
\hline Dystroglycan & $\mathrm{N} 494 \mathrm{H}$ & 33 & Homozygote $\mathrm{HH}$ à risque \\
\hline L-selectin & Pro213Ser & 125 & Allèle Ser à risque \\
\hline \multirow[t]{2}{*}{ VEGF } & $-460 \mathrm{~T} / \mathrm{C}$ & 181 & Allèle T à risque \\
\hline & $405 \mathrm{G} / \mathrm{C}$ & & Aucune \\
\hline \multirow[t]{4}{*}{ ACE } & Insertion (I) / délétion (D) & 245 & Aucune \\
\hline & 287 bp alu repeat intron 16 & 111 & Allèle $D$ à risque \\
\hline & & 192 & Aucune \\
\hline & & 1209 & Aucune \\
\hline ATR & $1166 \mathrm{~A} / \mathrm{C}$ & 1168 & Aucune \\
\hline GST & Val105lle & 133 & Isoforme lle à risque \\
\hline $\mathrm{m \varepsilon PHx}$ & Tyrl13His & & Aucune \\
\hline IGF-1 & $\begin{array}{l}\text { Nombre de «CA repeats» } \\
\text { dans le promoteur }\end{array}$ & 181 & Aucune \\
\hline IGF-IR & $3174 \mathrm{G} / \mathrm{A}$ & 132 & Aucune \\
\hline MTHFR & $677 \mathrm{C} / \mathrm{T}$ & 181 & Aucune \\
\hline \multirow[t]{2}{*}{ Factor VII } & $323 \mathrm{del} /$ ins & 1179 & Allèle ins protecteur \\
\hline & & 192 & Aucune \\
\hline \multirow[t]{2}{*}{ Factor XIII } & Val34Leu & 1179 & Aucune \\
\hline & & 192 & Aucune \\
\hline HLA & Antigène A2 & 77 & $\begin{array}{l}\text { Augmentation du risque de } \\
\text { DBP }\end{array}$ \\
\hline Urokinase & 3'UTR C/T & 204 & Aucune \\
\hline TAPl & Dpnll & 224 & Aucune \\
\hline
\end{tabular}

Tableau I. Études d'association gènes candidats dans la DBP. VEGF : vascular endothelial growth factor; $A C \varepsilon$ : angiotensin converting enzyme ; ATR : angiotensin receptor; GST : glutathione S-tranferase; mEPHx: microsomal epoxyde hydrolase ; IGF-1 : insulin growth factor 1 ; IGF-IR : insulin growth factor 1 receptor; MTHFR : 5, 10-methylenetetrahydrofolate reductase; HLA: human leukocyte antigen; TAPl: transporter associated with antigen processing; TNF $\alpha$ : tumor necrosis factor alpha; MBL2 : mannose binding lectin 2; IL: interleukin; $\mathrm{MCP} 1$ : monocyte chemoattractant protein 1; TGF $\beta$ : transforming growth factor beta; IFN $\gamma$ : interferon gamma; MIF : migration inhibitory factor; SP-B : surfactant protein B; SP-Al : surfactant protein Al.

considérablement au cours du développement alvéolaire chez le rat, suggérant son rôle au cours de l'alvéolisation [7]. Un autre gène prometteur a été identifié dans une des deux populations ethniques initiales, il s'agit du gène MMP16 (matrix metalloproteases) [8], qui d'une part intervient dans une voie de signalisation commune à la famille des gènes SPOCK [9], et d'autre part est très similaire à MMP14 dans sa structure et sa fonction, cette dernière jouant un rôle clé dans l'activation de MMP2 et dans le développement alvéolaire [10-12].

\section{Conclusion}

L'existence d'une prédisposition génétique à la DBP a été clairement établie par les études de concordance entre jumeaux. Cependant, comme dans toute pathologie multifactorielle, l'intervention des facteurs génétiques est complexe et la pathogénie de la maladie résulte d'interactions entre ces facteurs génétiques et les facteurs environnementaux. Les études « gènes candidats » publiées au cours de ces dernières années ont mis en évidence un certain nombre de molécules, mais dont les associations avec la DBP n'ont pas pu être confirmées dans de plus larges cohortes. Les résultats obtenus à l'issue de la première étude 
pangénomique démontrent le potentiel de ce type de stratégie pour identifier des gènes associés à la DBP, qui n'auraient pas été sélectionnées dans une approche candidate, mais qui ont un impact important sur le phénotype et sont fortement exprimés dans le poumon néonatal. À terme, l'identification de marqueurs génétiques fortement associés à la survenue d'une DBP doit permettre non seulement de mieux comprendre la physiopathologie de cette maladie, mais également de dégager de nouvelles pistes thérapeutiques. L'identification d'une voie de signalisation participant au risque de DBP peut faire envisager des thérapeutiques ciblant cette voie. Plus finement, l'identification de SNP, suivie de la mise en évidence de leur fonction sur l'expression du gène, peut permettre d'espérer une modulation thérapeutique directe de l'expression de ces mêmes gènes. $\diamond$

Bronchopulmonary dysplasia and genetics

\section{LIENS D'INTÉRÊT}

Les auteurs déclarent n'avoir aucun lien d'intérêt concernant les données publiées dans cet article.

\section{RÉFÉRENCES}

1. Zeitlin J, Ancel Py, Delmas D, et al. Changes in care and outcome of very preterm babies in the Parisian region between 1998 and 2003. Arch Dis Child Fetal Neonatal Ed 2010 ; 95 : F188-93.

2. Bhandari V, Gruen JR. The genetics of bronchopulmonary dysplasia. Semin Perinatol 2006 ; $30: 185-91$.

3. Parker RA, Lindstrom DP, Cotton RB. Evidence from twin study implies possible genetic susceptibility to bronchopulmonary dysplasia. Semin Perinatol 1996 ; $20: 206-9$.

4. Bhandari V, Bizzarro MJ, Shetty A, et al. Familial and genetic susceptibility to major neonatal morbidities in preterm twins. Pediatrics 2006 ; 117 : 1901-6.

5. Lavoie PM, Pham C, Jang KL. Heritability of bronchopulmonary dysplasia, defined according to the consensus statement of the national institutes of health. Pediatrics $2008 ; 122:$ 479-85.

6. Ehrenkranz RA, Walsh MC, Vohr BR, et al. Validation of the National Institutes of Health consensus definition of bronchopulmonary dysplasia. Pediatrics 2005 ; 116 : 1353-60.

7. Hadchouel A, Durrmeyer X, Bouzigon $\varepsilon$, et al. Identification of SPOCK2 as a susceptibility gene for bronchopulmonary dysplasia. Am J Respir Crit Care Med $2011 ; 184: 1164-70$.

8. Hadchouel A, Decobert F, Franco-Montoya ML, et al. Matrix metalloproteinase gene polymorphisms and bronchopulmonary dysplasia: identification of MMP16 as a new player in lung development. PLoS One 2008 ; 3: e3188.

9. Nakada M, Miyamori H, Yamashita J, Sato H. Testican 2 abrogates inhibition of membrane-type matrix metalloproteinases by other testican family proteins. Cancer Res 2003 ; 63 : 3364-9.

10. Atkinson JJ, Holmbeck K, Yamada S, et al. Membranetype 1 matrix metalloproteinase is required for normal alveolar development. Dev Dyn 2005 ; 232 : 1079-90.

11. Boucherat O, Bourbon JR, Barlier-Mur AM, et al. Differential expression of matrix metalloproteinases and inhibitors in developing rat lung mesenchymal and epithelial cells. Pediatr Res $2007 ; 62: 20-5$

12. Oblander $S A$, Zhou Z, Galvez BG, et al. Distinctive functions of membrane type 1 matrix-

metalloprotease (MT1-MMP or MMP-14) in lung and submandibular gland development are independent of its role in pro-MMP-2 activation. Dev Biol 2005 ; 277 : 255-69.

13. Zana-Taïeb $\varepsilon$, Jarreau P. Retard de croissance intrautérin et dysplasie broncho-pulmonaire. Med Sci (Paris) $2013 ; 29: 826-8$.

14. Lopez $\varepsilon$, Jarreau PH. Inflammation et dysplasie bronchopulmonaire. Med Sci (Paris) 2013 ; 29 : 823-5.

\section{NOUVELle}

\section{Inflammation et dysplasie bronchopulmonaire}

Emmanuel Lopez, Pierre-Henri Jarreau

> La dysplasie bronchopulmonaire (DBP) est la conséquence d'agressions pulmonaires multiples sur un poumon en développement $[1, \quad(\rightarrow)$ Voir les Nouvelles 2] $(\rightarrow)$.

La présence d'une réponse inflamde A. Hadchouel et C. Delacourt, $\varepsilon$. Zana-Taïeb et P.H. Jarreau, matoire dans les pages 821 et 826 de voies aériennes est ce numéro constante au cours de la DBP et apparaît comme une voie finale commune aux différents facteurs de risque [3-6]. $\varepsilon n$ anténatal, une chorioamniotite (CA) - qui est une inflammation du placenta et des membranes généralement due à une infection et fréquemment en cause dans les naissances prématurées - puis, en postnatal, la toxicité de l'oxygène, la ventilation mécanique et l'infection, entraînent une réponse inflammatoire pulmonaire responsable d'une inhibition de l'alvéolisation et de l'angiogenèse dans le poumon immature du nouveauné prématuré (Figure 1).

\section{Effets de l'inflammation anténatale sur le développement pulmonaire} De nombreuses études ont recherché une association entre chorioamniotite et DBP, mais les résultats sont contradictoires.
Service de médecine et réanimation néonatales de Port-Royal, Hôpital Cochin, 53, avenue de l'Observatoire, 75014 Paris, France ; Inserm U767, Fondation PremUP, Paris, France.

emmanuel.lopez@cch.aphp.fr

Des modèles animaux de chorioamniotite ont permis d'étudier les effets d'une inflammation anténatale sur le poumon fœtal. L'injection intraamniotique de lipopolysaccharide (LPS) d'Escherichia coli chez la brebis gestante entraîne un afflux de cellules inflammatoires et une production de cytokines pro-inflammatoires dans le chorion et le liquide amniotique, caractérisant la chorioamniotite. Cette réponse inflammatoire est également présente dans le poumon fœtal ; elle y est associée à une diminution de la septation alvéolaire (qui aboutit à la production de nouvelles alvéoles) et de 